\title{
Response bias in relational reasoning
}

\author{
STEPHEN E. NEWSTEAD and PAUL POLLARD \\ Plymouth Polytechnic, Plymouth, Devon, England \\ and \\ RICHARD A. GRIGGS \\ University of Florida, Gainesville, Florida
}

\begin{abstract}
In this study, we investigated the possibility that a response bias might be responsible for the typical pattern of responding observed when people reason about artificial relationships, especially set inclusions. Experiment 1 provided strong evidence for the proposed bias, since subjects tended to respond as if an unspecified relationship were symmetrical and increasingly intransitive over inferential distance. However, Experiment 2, using extended syllogisms, showed that not all relationships lead to such responding. Although the majority of the quantified relationships were responded to as if they were symmetrical, most were also regarded as transitive. Thus, the response bias idea, although of some interest, cannot provide a complete explanation of performance on these tasks.
\end{abstract}

In the literature on reasoning, there is a longstanding dispute between the rationalists and nonrationalists. The rationalists believe that reasoning is inherently logical and that errors can be explained by misinterpretation of the premises, whereas the nonrationalists believe that errors can be attributed to nonlogical response biases.

In the present paper, we attempt to make a contribution to this dispute by exploring the existence of a nonlogical response bias that may explain performance on a variety of reasoning tasks involving artificial relationships. The bias in question is a tendency to assume that any relationship is (1) symmetrical and (2) increasingly intransitive over inferential distance. This can be illustrated best by considering the schematic relationship A-B, B-C, C-D, where A, B, C, and D are entities and "." depicts any relationship connecting them. To say that the relationship is symmetrical means that subjects will assume that the relationship holds both ways, so that, for example, B-A is true as well as A-B. To say that the relationship is intransitive implies that transitive inferences will not tend to be accepted as true: Subjects will tend not to accept conclusions such as A-C or B-D. To say that the relationship is increasingly intransitive over inferential distance means that as the number of steps required to make a transitive inference increases, subjects will be less likely to accept the inference. Thus, in the above example, A-D would be less likely to be accepted than A-C or B-D.

Although such a bias has not been discussed previously in the literature, there is in fact a range of evidence consistent with it. One line of evidence comes from Tsal (1977), who gave subjects problems involving a relation-

The authors thank Ray Burke for his assistance in generating the experimental materials for Experiment 2. Requests for reprints should be sent to S. E. Newstead, Department of Psychology, Plymouth Polytechnic, Drake Circus, Plymouth, Devon PL4 8AA, England. ship among four people, but the actual nature of the relationship was not specified (it was denoted by a short line, as in the schematic example above). The majority of subjects $(\mathbf{7 2 \%})$ assumed that the relationship was symmetrical, and $76 \%$ assumed it was intransitive. Because transitivity was investigated only at one inferential step, it is impossible to determine whether intransitivity increased over inferential distance. Assumptions of symmetry have also been reported by DeSoto and Kuethe (1959) with interpersonal relationships, and Wason and Johnson-Laird (1972) reported a variety of experiments indicating that subjects assume that conditional statements are symmetrical.

However, perhaps the best evidence for the bias comes from work on artificial set inclusions or extended syllogisms (e.g., Griggs, 1976). A four-term set inclusion consists of the information that all As are Bs, all Bs are Cs, and all Cs are Ds. Such a relationship is antisymmetrical, in the sense that one cannot conclude with certainty that all Bs are As (although this would be the case if A and $B$ were identical sets). Nevertheless, there is evidence that many subjects assume symmetry. The relationship is perfectly transitive, in that one can conclude that all As are $C s$, all Bs are Ds, and all As are Ds. Nevertheless, subjects seem reluctant to draw such transitive inferences, and this reluctance increases over inferential distance, as illustrated by the classic distance $\times$ truth interaction, which is almost invariably found when performance on artificial set inclusions is studied (e.g., Griggs, 1976).

All previous explanations of set inclusion performance can be loosely classified as interpretational. Potts (1978) suggested that set inclusions were misinterpreted as similarity relationships; Mynatt and Smith (1979) suggested that they cued in an inappropriate processing schema; and Newstead and Griggs (1984) argued that er- 
rors might result from the quantifier all being interpreted fuzzily and thus allowing the occasional exception. None of these interpretational explanations has attracted widespread support, so it seems reasonable to explore in more detail the explanation in terms of response bias. The purpose of Experiment 1 was to investigate whether the response bias exists. The Tsal technique, in which terms are linked by an unknown relationship depicted by a line, was used. If the response bias exists, then the relationship should be assumed to possess the properties of intransitivity increasing over inferential distance and symmetry.

\section{EXPERIMENT 1}

\section{Method}

Subjects. Fifty-four first-year psychology students from Plymouth Polytechnic participated in the experiment for course credit.

Materials. Three different passages were used, one each involving people's names, letters of the alphabet, and a tribe called the Fundalas. This was the Fundalas passage:

This passage describes the relationship between the Fundalas (a primitive tribe), outcasts, hill people, and farmers.

Fundalas-Outcasts

Outcasts-Hill people

Hill people-Farmers

The other passages were similar in that they used four terms, and the relationship between terms was denoted by a line. Beneath each passage were 12 test items, presented in random order. Three items were forward adjacents (e.g., Hill people-Farmers); these simply repeat the original information in the same direction as it was presented. Three were converted adjacents (e.g., Farmers-Hill people), which are the reversed forms of the original information. Two were forward remotes at Distance 1 (e.g., Fundalas-Hill people), and two were converted remotes at Distance 1. One was a forward remote at Distance 2 (Fundalas-Farmers), and one was the converted version of this. (Remoteness indicates how many other terms intervened between the two terms in the test item.) Next to each test item were the letters $T$ and F.

Subjects were told that the passage described an unknown relationship between four entities; their task was to indicate, by circling the $\mathrm{T}$ or $\mathrm{F}$, whether each of the statements given was true or false. True statements were defined as those that occurred in the passage or could be logically inferred from it; all other statements were defined as being false.

Procedure. Subjects were run in a single group. Each subject received a booklet containing the instructions plus the three passages and was allowed as much time as needed to finish the booklet. The order of the passages was randomized for each subject.

\section{Results and Discussion}

The results are presented in Table 1 in terms of the number of "true" responses given to the various types of test item. The factors in the analysis of variance were type of passage (people's names vs. letters vs. Fundalas), direction (forward vs. converted), and distance ( 0 vs. 1 vs. $2)$. The type of passage had no significant effect $(F<1)$, and it did not interact with any of the other factors. Hence, the data in Table 1 are collapsed over this factor.

There was a main effect of direction $[F(1,53)=7.74$, $p<.01$ ], with forward items producing more true responses $(47 \%)$ than did converted items $(40 \%)$. There was also a main effect of distance $[F(2,106)=197.3$, $p<.001]$, with adjacent items producing more true responses $(89 \%)$ than did those at Distance $1(27 \%)$,
Table 1

Proportion of "True" Responses in Experiment 1 as a Function of Direction and Distance

\begin{tabular}{lccc}
\hline & \multicolumn{3}{c}{ Distance } \\
\cline { 2 - 4 } Direction & 0 & 1 & 2 \\
\hline Forward & $.97(.98)$ & $.31(.33)$ & $.13(.27)$ \\
Converted & $.82(.79)$ & $.24(.25)$ & $.13(.19)$ \\
\hline
\end{tabular}

Note-The data in parentheses are from the replication experiment.

which in turn produced more true responses than did those at Distance $2(13 \%)$. Both of these differences were significant $[t(53)=15.14, p<.001$ and $t(53)=3.55$, $p<.001$, respectively]. In addition, there was a significant interaction between direction and distance $[F(2,106)$ $=10.98, p<.001]$. There was a difference between forward and converted items at Distance 0 which was less at Distance 1 and not present at Distance 2 .

The results of this experiment are quite clear-cut. First, the majority of subjects (over $80 \%$ ) responded as if the relationship were symmetrical, since they called converted adjacents true. Second, subjects responded as if the relationship were intransitive and increasingly so over inferential distance. For both forward and converted items, there were fewer "true" responses at Distance 2 than at Distance 1 . Both of these differences were significant [ $t(53)$ $=4.38, p<.001$ and $t(53)=2.72, p<.01]$.

However, it is possible that these results may be artifacts of the technique used. For this reason, we decided to repeat the experiment using different modes of presentation, in one condition putting all the premises on a single line of text, and in another linking the items by an asterisk instead of a line. The mode of presentation had no effect on the results $(F<1)$. The findings are given in parentheses in Table 1, from which it can be seen that the results were very similar to those obtained in Experiment 1 ; hence the pattern of results is clearly consistent over different subjects and different ways of presenting the relationship.

Although the results of Experiment 1 provide evidence for the existence of the response bias, it would be premature to conclude that this can explain set inclusion performance. The aim of Experiment 2 was to obtain further evidence on this issue by examining performance with other relationships. Extended syllogisms using a wide range of quantifiers were used to test the prediction that these will produce performance consistent with the response bias.

The quantifiers we investigated were: all, most, many, some, several, a few, some...not, and none. The logically correct response pattern for the quantifiers used in classical syllogisms is straightforward: all is transitive and antisymmetrical; some is intransitive and symmetrical; some...not is intransitive and asymmetrical; and none is intransitive and symmetrical. Most and many are intransitive and asymmetrical. Several and few are intransitive, but it is a moot point as to whether they are symmetrical. It depends on whether they are interpreted as implying a constant proportion or a specific number. Since Borges 
and Sawyers (1974) have reported evidence in favor of constant proportionality, it will be assumed here that several and $a$ few are asymmetrical.

\section{EXPERIMENT 2}

\section{Method}

Subjects. The 256 subjects were undergraduates at the University of Florida who participated to help fulfill an introductory course requirement.

Materials. Computer-generated sheets were used to present the material. Each sheet contained the instructions (which were identical to those used in Experiment 1), followed by the passage, followed by the test items. The passage involved five terms and either described the relationship between letters of the alphabet or used the Fundalas material. The 20 test items appeared in random order, each followed by a $\mathrm{T}$ or $\mathrm{F}$. The same quantifier (all, most, many, some, several, a few, some...not, or none) appeared in the passage and the test items. The use of five-term relationships as opposed to the four-term ones previously used enabled a closer examination of the effects of inferential distance.

Procedure. Subjects were run in groups of varying sizes. Each subject received only one sheet. Since there were eight quantifiers and two types of content with each quantifier, there were 16 experimental groups with 16 subjects in each of them. Subjects were allowed as much time as they required to complete the experiment.

\section{Results and Discussion}

The results are summarized in Table 2. The data are presented in terms of the proportion of "true" responses given to forward and converted items. For ease of presentation, the results in this table have been collapsed over the different kinds of materials. The results were analyzed using a four-way analysis of variance in which the factors were quantifier used, materials (letters vs. Fundalas), direction (forward vs. converted) and distance $(0,1,2$, or 3). For reasons of clarity, the results will be presented in a number of sections.

Symmetry. Overall, there was a stronger tendency to respond "true" to forward items than to converted items $[F(1,240)=141.26, p<.001]$. Nevertheless, there was good evidence for the symmetric response bias since subjects responded "true" to simple converted items (i.e., those at Distance 0) on 59\% of the trials. Because this response was definitely correct for only two of the eight quantifiers (some and none), this seems to provide fairly

Table 2

Proportion of True Responses Produced in Experiment 2 as a Function of Quantifier, Direction, and Distance

\begin{tabular}{|c|c|c|c|c|c|c|c|c|}
\hline \multirow[b]{2}{*}{ Quantifiers } & \multicolumn{4}{|c|}{ Distance } & \multicolumn{4}{|c|}{ Distance } \\
\hline & 0 & 1 & 2 & 3 & 0 & 1 & 2 & 3 \\
\hline & \multicolumn{4}{|c|}{ Forward Items } & \multicolumn{4}{|c|}{ Converted Items } \\
\hline All & $.97^{\mathrm{T}}$ & $.89^{\mathrm{T}}$ & $.88^{\mathrm{T}}$ & $.81^{\mathrm{T}}$ & $.38^{\mathrm{F}}$ & $.40^{\mathrm{F}}$ & $.34^{\mathrm{F}}$ & $.34^{\mathrm{F}}$ \\
\hline Most & $1.00^{\mathrm{T}}$ & $.77^{\mathrm{F}}$ & $.64^{\mathrm{F}}$ & $.69^{F}$ & $.29^{\mathrm{F}}$ & $.29^{\mathrm{F}}$ & $.23^{\mathrm{F}}$ & $.19^{\mathrm{F}}$ \\
\hline Many & $.96^{\mathrm{T}}$ & $.77^{\mathrm{F}}$ & $.64^{\mathrm{F}}$ & $.69^{\mathrm{F}}$ & $.50^{\mathrm{F}}$ & $.52^{\mathrm{F}}$ & $.44^{\mathrm{F}}$ & $.44^{\mathrm{F}}$ \\
\hline Some...Not & $.99^{\mathrm{T}}$ & $.61^{\mathrm{F}}$ & $.56^{\mathrm{F}}$ & $.56^{\mathrm{F}}$ & $.54^{\mathrm{F}}$ & $.34^{\mathrm{F}}$ & $.30^{\mathrm{F}}$ & $.22^{F}$ \\
\hline Some & $.99^{\mathrm{T}}$ & $.77^{\mathrm{F}}$ & $.70^{\mathrm{F}}$ & $.66^{\mathrm{F}}$ & $.89^{\mathrm{T}}$ & $.66^{\mathrm{F}}$ & $.58^{\mathrm{F}}$ & $.56^{\mathrm{F}}$ \\
\hline Several & $.97^{\mathrm{T}}$ & $.71^{\mathrm{F}}$ & $.67^{\mathrm{F}}$ & $.59^{\mathrm{F}}$ & $.72^{\mathrm{F}}$ & $.54^{\mathrm{F}}$ & $.44^{F}$ & $.41^{\mathrm{F}}-\left({ }^{2}\right.$ \\
\hline A few & $.98^{\mathrm{T}}$ & $.71^{\mathrm{F}}$ & $.70^{\mathrm{F}}$ & $.59^{\mathrm{F}}$ & $.66^{\mathrm{F}}$ & $.53^{\mathrm{F}}$ & $.48^{\mathrm{F}}$ & $.59^{\mathrm{F}}$ \\
\hline None & $.97^{\mathrm{T}}$ & $.26^{\mathrm{F}}$ & $.20^{\mathrm{F}}$ & $.28^{\mathrm{F}}$ & $.78^{\mathrm{T}}$ & $.25^{\mathrm{F}}$ & $.19^{\mathrm{F}}$ & $.22^{\mathrm{F}}$ \\
\hline Mean & .98 & .69 & .62 & .61 & .59 & .44 & .38 & .37 \\
\hline
\end{tabular}

Note-Superscripts indicate the correct response. good evidence of an overall tendency to assume symmetry. It should be noticed, however, that the frequency of "true" responses to converted items varied with the quantifier used, manifested in an interaction between quantifier and direction $[F(7,240)=6.78, p<.001]$. At Distance 0, the two quantifiers producing the most "true" responses were some and none, precisely those quantifiers for which "true" is the correct response. Hence, although there was evidence consistent with the response bias explanation, there was also good evidence for responding in accordance with logic.

Transitivity. Inspection of performance on forward items at Distance 1 reveals that on $69 \%$ of the trials, subjects responded as if the quantifier were transitive. This was surprisingly high with respect to the results of Experiment 1 , since in that study, transitivity was indicated on only $31 \%$ of the trials for Distance 1 . It was also high with respect to logic, since only one of the quantifiers (all) possesses this property. Furthermore, previous research using the quantifier all has typically produced poorer performance on transitive items.

The ANOVA revealed a main effect of distance $[F(3,720)=94.40, p<.001]$. Each pairwise comparison was significant except that between Distances 2 and 3 . There was an interaction between distance and direction $[F(3,720)=13.25, p<.001]$; but the distance effect was still clearly marked for both forward and converted items.

Overall there is evidence for transitivity, and it appears to decline over inferential distance. It would appear, however, that there is no further decline after Distance 2.

Differences between quantifiers. There was a main effect of quantifier $[F(7,240)=5.60, p<.001]$. Furthermore, as already indicated, there was an interaction between quantifier and direction, and also between quantifier and distance $[F(21,720)=5.44, p<.001]$. Clearly subjects were not treating all the quantifiers in the same way, as would have occurred if they were responding purely on the basis of the response bias. In fact, the differences between quantifiers do by and large reflect a tendency to respond in accordance with logic, as was indicated earlier.

Strategies. Another way of looking at the data from this experiment is in terms of the strategies that subjects adopted (classified according to the criteria suggested by Mynatt \& Smith, 1979). The results illustrate quite forcibly the points that have already emerged: strategies involving symmetry were more frequent than those involving asymmetry, and strategies involving transitivity were more frequent than those involving intransitivity. Most strikingly, the occurrence of the strategy predicted by the response bias-symmetry and intransitivity-was very rare.

\section{GENERAL DISCUSSION}

The results of Experiment 2 are somewhat disappointing with respect to the response bias theory. Although there was evidence for assumptions of symmetry with most of the quantifiers, intransitivity was not 
indicated, although there was evidence for decreasing transitivity over inferential distance. While the response bias may make a contribution to the results, it is clear that other (presumably interpretational) factors are also involved. There was good evidence in the performance on the different quantifiers for responding in accordance with logic. The present experiments clearly indicate the problems and pitfalls that are present in research designed to distinguish empirically between rationalist and nonrationalist theories.

\section{REFERENCES}

Borges, M. A., \& SAWYers, B. K. (1974). Common verbal quantifiers: Usage and interpretation. Journal of Experimental Psychology, 102, 335-338.

DeSoto, C. B., \& KuETHE, J. L. (1959). Subjective probabilities of interpersonal relationships. Journal of Abnormal \& Social Psychology, 59, 290-294.
GrIGGS, R. A. (1976). Logical processing of set inclusion relations in meaningful text. Memory \& Cognition, 4, 730-740.

MynatT, B. T., \& SMITH, K. H. (1979). Processing of text containing artificial inclusion relations. Memory \& Cognition, 7, 390-400.

Newstead, S. E., \& Griggs, R. A. (1984). Fuzzy quantifiers as an explanation of set inclusion performance. Psychological Research, 46, 377-388.

PotTs, G. R. (1978). The role of inference in memory for real and artificial information. In R. Revlin \& R. E. Mayer (Eds.), Human reasoning (pp. 139-161). Washington, DC: Winston.

TSAL, Y. (1977). Symmetry and transitivity assumptions about a nonspecified logical relation. Quarterly Journal of Experimental Psychology, 29, 677-684.

Wason, P. C., \& Johnson-Laird, P. N. (1972). Psychology of reasoning: Structure and content. London: Batsford.

(Manuscript received for publication November 25, 1985.) 